\title{
Relationship between dietary patterns and risk factors for cardiovascular disease in patients with type 2 diabetes mellitus: a cross-sectional study
}

\author{
Yusuke Osonoi ${ }^{1}$, Tomoya Mita ${ }^{1,2^{*}}$, Takeshi Osonoi ${ }^{5}$, Miyoko Saito $^{5}$, Atsuko Tamasawa ${ }^{5}$, Shiho Nakayama ${ }^{1}$, \\ Yuki Someya ${ }^{1}$, Hidenori Ishida ${ }^{5}$, Akio Kanazawa ${ }^{1,3}$, Masahiko Gosho ${ }^{6}$, Yoshio Fujitani ${ }^{1}$ and Hirotaka Watada ${ }^{1,2,3,4}$
}

\begin{abstract}
Background: While some dietary patterns are associated with the incidence of type 2 diabetes mellitus (T2DM) and cardiovascular disease (CVD), the relationship between dietary pattern and risk factors for CVD in patients with T2DM remains to be clarified. The aim of this study was to identify dietary patterns and investigate the relationship between dietary patterns and potential risk factors for CVD in patients with T2DM.

Methods: The study participants comprised 726 Japanese T2DM outpatients free of history of CVD. Life styles were analyzed using self-reported questionnaires. The relationship between dietary patterns, identified by factor analysis, and potential risk factors for CVD was investigated by linear and logistic regression analyses.

Results: Six dietary patterns were identified by factor analysis. Especially, three dietary patterns were associated with risk factors for CVD. The "Seaweeds, Vegetables, Soy products and Mushrooms" pattern, characterized by high consumption of seaweeds, soy products and mushrooms, was associated with lower use of diabetes medication and healthier lifestyles. The "Noodle and Soup" pattern, characterized by high consumption of noodle and soup was associated with higher body mass index, alanine aminotransferase, aspartate aminotransferase, $\gamma$-glutamyl transpeptidase and triglyceride levels. The "Fruit, Dairy products and Sweets" pattern was associated with lower Y-glutamyl transpeptidase levels, blood pressure, albuminuria and brachial-ankle pulse wave velocity.
\end{abstract}

Conclusions: The findings suggested that dietary patterns correlated with risk factors for CVD in T2DM patients.

Keywords: Dietary pattern, Type 2 diabetes mellitus, Risk factors for cardiovascular disease, Self-reported questionnaires

\section{Background}

The onset of type 2 diabetes mellitus (T2DM) is associated with numerous lifestyle problems. Indeed, multiple lifestyle modifications, in addition to pharmacological intervention for classical risk factors, can reduce both the incidence of T2DM [1] in non-T2DM population and the development of cardiovascular disease (CVD) in patients with T2DM [2, 3]. However, a recent clinical trial showed that life style intervention, with a special

\footnotetext{
* Correspondence: tom-m@juntendo.ac.jp

'Department of Metabolism \& Endocrinology, Juntendo University Graduate School of Medicine, 2-1-1 Hongo, Bunkyoku, Tokyo 113-8421, Japan

${ }^{2}$ Center for Molecular Diabetology, Juntendo University Graduate School of Medicine, Bunkyoku, Tokyo 113-8421, Japan

Full list of author information is available at the end of the article
}

focus on reduced calorie intake and increased physical activity, did not affect the rate of CVD in obese patients with T2DM [4]. In this regard, it is possible that not only caloric intake, but rather dietary composition, may need to be modified to achieve appropriate metabolism and prevent or delay CVD in patients with T2DM.

Recent studies showed that specific foods such as fruit and vegetables [5], nutritional supplements, such as magnesium and calcium [6-8], and quality of carbohydrate intake, such as high fibers, low glycemic index and whole grain intake [8-10], were associated with lower risk of T2DM. However, in real life, people do not consume certain foods or single nutrients, but rather mixed food that contains various nutrients. In this regard, the 
dietary pattern should be taken into consideration, because it reflects the complexity of dietary intake, where the food contents usually have interactive and synergistic effects and occasionally even antagonistic effects [11]. In fact, it was suggested that dietary patterns may be more predictive of disease risks compared to specific foodand nutrient-based approaches [11].

Recent studies demonstrated that healthy Asian dietary patterns characterized by high consumption of vegetables, fruits, seaweeds, bonefishes, potatoes and/or soy foods were inversely associated with incidence of T2DM [12-14], metabolic syndrome [15], atherosclerosis [16] and related CVD [17] in non-T2DM general population, while western dietary pattern was associated with high incidence of T2DM $[10,18,19]$ and metabolic syndrome [20]. Although it was demonstrated that the consumption of fruits, vegetable foods and meat was higher in patients with T2DM than non-T2DM subjects [21], only a few studies demonstrated that Korean Healthy diet pattern characterized by whole grains, legumes, vegetables and fruits was inversely associated with lipid metabolism [22], and vegetable and fish pattern was associated with better renal function in patients with T2DM [23]. Thus, there seems to be some gaps in our understanding of the relationship between dietary patterns and risk factors for CVD, especially arterial stiffness, in patients with T2DM. The aim of this crosssectional study was to assess the above relationship in Japanese patients with T2DM free of history of CVD

\section{Research design and methods}

\section{Subjects}

The subjects of this cohort study were recruited from the Diabetes Outpatient Clinic of Juntendo University (Tokyo, Japan), Naka Memorial Clinic (Naka, Japan), and Secomedic Hospital (Funabashi, Japan) as reported previously [24]. Briefly, the inclusion criteria were as follows: 1) T2DM patients, 2) $\geq 25$ years of age and $<70$ years of age (regardless of gender), and 3) signing consent form for participation in the study. The following exclusion criteria were also applied: 1) type 1 or secondary diabetes, 2) presence of severe infectious disease, before or after surgery, or severe trauma, 3) history of myocardial infarction, angina pectoris, cerebral stroke, or cerebral infarction, 4) chronic renal failure requiring hemodialysis, 5) liver cirrhosis, 6) moderate or severe heart failure (NYHA/New York Heart Association stage III or higher), 7) active malignancy, 8) pregnant, lactating, or possibly pregnant women, or those planning to become pregnant during the study period, 9) patients judged as ineligible by the clinical investigators.

A total of 1,032 consecutive subjects were screened between June 2013 and January 2014. Among them, 906 patients who met the above eligibility criteria were invited to participate in the present study. After providing information on the purpose and procedures of the study, 736 patients with T2DM accepted the invitation and were enrolled in this study. The study was approved by the Institutional Review Board of Juntendo University Hospital and conducted in accordance with the principles described in the Declaration of Helsinki. All patients provided written informed consent prior to participation. The study was registered on the University Hospital Medical Information Network Clinical Trials Registry (UMIN000010932).

\section{Questionnaire survey}

Questionnaire survey was conducted using valid and reliable self-administered questionnaires described previously [24]. Briefly, dietary habits during the preceding month were assessed with the validated, Brief, selfadministered Diet History Questionnaire (BDHQ). The BDHQ is a 4-page structured questionnaire that asks about consumption and frequency of selected foods to estimate the dietary intake of 56 food and beverage items with specified serving size described in terms of consumption in general Japanese populations [25].

We also used the Morning Evening Questionnaire (MEQ) [26], which is a self-assessment questionnaire developed primarily for screening candidates for sleep-related experiments to evaluate morningness and eveningness in individuals. A high MEQ score represents morning type.

The Pittsburg Sleep Quality Index (PSQI) [27] is a selfadministered questionnaire designed to evaluate sleep quality and consists of 18 items that in turn are comprised of 7 components, which include subjective sleep quality, sleep duration, sleep onset, habitual sleep efficiency, sleep disturbances, use of sleeping medications, and daytime dysfunction, with each weighted equally on a $0-3$ scale, to be summed to yield the global PSQI score ranging from 0 to 21 , where the higher the scores, the worse the sleep quality. The PSQI has a high test-retest reliability and a good validity [28].

The participating patients also completed the BDI (Beck Depression inventory)-II, which is a 21-item questionnaire that assesses hopelessness, irritability, cognition, guilt, fatigue, weight loss, and sexual interest, representing depression-related symptoms in adults and adolescents [29]. A high BDI-II score represents depressive state.

Physical activity level was assessed with the International Physical Activity Questionnaire (IPAQ) that comprises four simple questions on physical activity [30]. The IPAQ results are expressed as metabolic equivalent scores $\left(\right.$ METs-hour-week ${ }^{-1}$ ).

Workers were defined as full-time employees or shift workers by a question in the questionnaire, as described previously [24]. The subjects were also divided into nonsmokers, former smokers or current smokers, as described previously [24]. 


\section{Blood and urine tests}

Blood samples were obtained at visits to the Outpatients Clinic after overnight fast. Liver and renal function tests, lipids, HbA1c (National Glycohemoglobin Standardization Program), and glucose were measured with standard techniques. UAE was measured by the latex agglutination assay using a spot urine sample. The estimated glomerular filtration rate (eGFR) was calculated by the formula: eGFR (ml/ min per $\left.1.73 \mathrm{~m}^{2}\right)=194 \times$ Age $^{-0.287} \times$ serum creatinine ${ }^{-0.1094}$ ( $\times 0.739$ for females), as described previously [24] .

\section{Measurement of baPWV}

baPWV was measured using an automatic waveform analyzer (BP-203RPE; Colin Medical Technology, Komaki, Japan), as described previously [24, 31]. Briefly, recording was performed with the patients in the supine position after resting for five minutes. Occlusion and monitoring cuffs were placed snugly around both areas in the upper and lower extremities. The pressure waveforms were then recorded simultaneously from the brachial arteries by the oscillometric method. All scans were automatically conducted by well-trained investigators who were blinded to the clinical information. The validity and reproducibility of baPWV measurements have been confirmed to be considerably high [32].

\section{Statistical analysis}

Results are presented as mean \pm SD or median (interquartile range: $25 \%$ to $75 \%$ ) for continuous variables or number (proportion) of patients for categorical variables. Some parameters were logarithmically transformed to approximate normal distribution. We used factor analysis with varimax rotation to reduce the complexity of dietary patterns of patients with T2DM based on the results of BDHQ. Factors with an eigenvalue $>1.25$ were retained [33]. Individual food items with a factor loading of $>|0.4|$ are highlighted as composing that factor for simplicity. The factor scores for each dietary pattern and for each subject were calculated by summing each dietary pattern score weighted by their factor loadings. The estimated factor scores were categorized into quintiles. Trend association across the quintile was evaluated by linear regression analysis for continuous variables or logistic regression analysis for categorical variables. We developed three models to evaluate the trend adjusted for age and gender or adjusted for age, gender, and body mass index (BMI), MEQ, PSQI, BDI-II, current smoking and physical activity. The model for eGFR was adjusted for BMI, MEQ, PSQI, BDI-II, current smoking and physical activity. Statistical tests were two-sided with $5 \%$ significant level. All analyses were performed using the SAS software version 9.3 (SAS Institute, Cary, NC).

\section{Results}

Among 736 patients, 10 patients did not complete the questionnaires, and were thus excluded from analysis.
The study participants comprised 726 Japanese patients with T2DM who were being treated on an outpatient basis. Table 1 lists the characteristics of the study subjects. The mean age was $57.8 \pm 8.6$ years, $62.9 \%$ male and $\mathrm{HbA} 1 \mathrm{c}$ was $7.0 \pm 1.0 \%$.

Factor analysis with varimax rotation identified six life style patterns (Table 2). Factor 1, with high loading for seaweeds, vegetables, soy products and mushroom, was labeled the "Seaweeds, Vegetables, Soy products and Mushrooms" pattern. Factor 2, which was characterized by fish, potatoes, meat and fats and oils, was labeled "Fish and Meat" pattern. Factor 3 seemed to load consumption of noodle and soup and thus it was named "Noodle and Soup" pattern. Factor 4 seemed to be characterized by meat, fats

Table 1 Patients characteristics $(n=726)$

\begin{tabular}{|c|c|}
\hline \multicolumn{2}{|l|}{ Demographic data } \\
\hline Age (years) & $57.8 \pm 8.6$ \\
\hline Gender (male) & $456(62.9)$ \\
\hline Estimated duration of diabetes (years) & $9.9 \pm 7.2$ \\
\hline Body mass index $\left(\mathrm{kg} / \mathrm{m}^{2}\right)$ & $24.6 \pm 4.1$ \\
\hline $\mathrm{HbA1c}(\%)$ & $7.0 \pm 1.0$ \\
\hline Fasting blood glucose (mg/dl) & $134 \pm 31$ \\
\hline Systolic blood pressure (mmHg) & $127 \pm 14$ \\
\hline Diastolic blood pressure (mmHg) & $77 \pm 11$ \\
\hline Total cholesterol (mg/dL) & $185 \pm 28$ \\
\hline High-density lipoprotein-cholesterol (mg/dL) & $59 \pm 14$ \\
\hline Triglyceride (mg/dL) & $100[70,152]$ \\
\hline Aspartate aminotransferase $(\mathrm{U} / \mathrm{L})$ & $21[18,27]$ \\
\hline Alanine aminotransferase $(\mathrm{U} / \mathrm{L})$ & $22[16,33]$ \\
\hline Y-glutamyl transpeptidase (U/L) & $25[17,39]$ \\
\hline Uric Acid (mg/dl) & $5.5 \pm 1.2$ \\
\hline Estimated glomerular filtration rate $\left(\mathrm{ml} / \mathrm{min} / 1.73 \mathrm{~m}^{2}\right)$ & $78 \pm 18$ \\
\hline Urinary albumin excretion (mg/g creatinine) & $10[6,23]$ \\
\hline brachial-ankle pulse wave velocity $(\mathrm{cm} / \mathrm{s})$ & $1543 \pm 279$ \\
\hline Morningness-Eveningness Questionnaire & $57.4 \pm 7.3$ \\
\hline Pittsburg Sleep Quality Index & $5.1 \pm 3.0$ \\
\hline Beck Depression inventory -\| & $9.9 \pm 7.6$ \\
\hline Energy intake (kcal/day) & $1713 \pm 582$ \\
\hline Physical activity (Mets/h/week) & $42.8 \pm 70.5$ \\
\hline Sleep duration (hours) & $6.4 \pm 1.2$ \\
\hline Current smoker (yes) & $174(24.0)$ \\
\hline Alcohol (g/day) & $12.3 \pm 21.5$ \\
\hline \multicolumn{2}{|l|}{ Treatment modality (n/\%) } \\
\hline Diabetes medication (yes) & $620(85.5)$ \\
\hline Hypertension medication (yes) & $346(47.7)$ \\
\hline Hyperlipidemia medication (yes) & $442(61.0)$ \\
\hline
\end{tabular}

Data are mean \pm SD or number (percentage) of patients 
Table 2 Food items and factor analysis with varimax rotation

\begin{tabular}{|c|c|c|c|c|c|c|}
\hline Components and item labels & Factor 1 & Factor 2 & Factor 3 & Factor 4 & Factor 5 & Factor 6 \\
\hline Rice & -0.07 & 0.07 & 0.00 & 0.06 & -0.15 & 0.80 \\
\hline Noodle & 0.07 & 0.14 & 0.96 & 0.04 & 0.02 & 0.05 \\
\hline Breads & 0.07 & -0.07 & 0.24 & 0.36 & 0.42 & -0.15 \\
\hline Miso soup & 0.18 & 0.02 & 0.08 & -0.06 & 0.09 & 0.81 \\
\hline Dairy products & 0.27 & -0.07 & -0.01 & 0.17 & 0.45 & 0.06 \\
\hline Meats & 0.03 & 0.52 & 0.06 & 0.52 & -0.09 & 0.02 \\
\hline Processed meats & 0.12 & 0.02 & 0.01 & 0.66 & 0.16 & -0.03 \\
\hline Fish and shellfish & 0.32 & 0.63 & 0.17 & 0.16 & -0.03 & 0.05 \\
\hline Processed fish & 0.23 & 0.60 & 0.10 & 0.16 & 0.07 & 0.14 \\
\hline Eggs & 0.20 & 0.32 & 0.01 & 0.41 & 0.03 & -0.01 \\
\hline Soy products & 0.63 & 0.10 & 0.13 & 0.13 & 0.13 & 0.08 \\
\hline Green \& dark yellow vegetables & 0.74 & 0.07 & -0.11 & 0.29 & 0.06 & -0.07 \\
\hline White vegetables & 0.73 & 0.25 & 0.11 & 0.13 & 0.00 & -0.07 \\
\hline Pickled vegetables & 0.51 & 0.18 & -0.03 & -0.07 & 0.04 & 0.10 \\
\hline Fruit and vegetable Juices & 0.04 & 0.16 & -0.01 & -0.01 & 0.02 & -0.09 \\
\hline Fruit & 0.21 & 0.35 & -0.01 & -0.03 & 0.56 & -0.05 \\
\hline Sugary foods & 0.15 & 0.51 & 0.00 & -0.05 & 0.20 & 0.21 \\
\hline Mushrooms & 0.55 & 0.41 & 0.01 & 0.01 & 0.13 & -0.14 \\
\hline Seaweeds & 0.70 & 0.06 & 0.07 & 0.05 & -0.02 & 0.10 \\
\hline Potatoes & 0.18 & 0.62 & 0.01 & 0.03 & 0.21 & -0.07 \\
\hline Sweets & -0.03 & 0.21 & 0.13 & 0.17 & 0.62 & 0.06 \\
\hline Fats and oils & -0.07 & 0.49 & 0.22 & 0.64 & -0.08 & 0.02 \\
\hline Alcohol & 0.01 & 0.02 & 0.20 & 0.17 & -0.53 & 0.15 \\
\hline Tea & 0.04 & 0.06 & 0.03 & 0.13 & 0.34 & 0.11 \\
\hline Coffee & 0.09 & 0.02 & 0.04 & 0.14 & 0.14 & 0.01 \\
\hline Soft drinks & -0.05 & -0.09 & 0.21 & 0.19 & 0.07 & 0.03 \\
\hline Seasonings & 0.32 & 0.05 & -0.09 & 0.60 & 0.03 & 0.06 \\
\hline Soup & 0.08 & 0.11 & 0.96 & 0.02 & 0.01 & 0.04 \\
\hline Contribution & $19 \%$ & $8 \%$ & $6 \%$ & $6 \%$ & $5 \%$ & $4 \%$ \\
\hline
\end{tabular}

Individual food items with a factor loading of $>|0.4|$ are shown in bold

and oils, seasonings and eggs, and was named "Meat, Fats and Oils, Seasonings and Eggs" pattern. Factor 5 seemed to be characterized by sweet, fruit and dairy products and was labeled "Fruit, Dairy products and Sweets" pattern. Factor 6 seemed to be characterized by rice and miso soups, and was named "Rice and Miso soups" pattern. Overall, these six diet patterns could account for $48 \%$ of the variance in food intake.

The characteristics across quintile of dietary pattern are shown in Tables 3, 4, 5 and 6. Table 3 shows the characteristics of the study subjects according to the quintiles of "Seaweeds, Vegetables, Soy products and Mushrooms" pattern scores in age and gender adjusted model. Subjects with a higher score for this pattern were older, more morning type, higher consumption of food, higher physical activity and less depressive status. While
HbA1c levels were comparable between the lowest quintile and the highest quintile, the lower prevalence of diabetes medication was found in the highest quintile in age- and gender-adjusted model. Subjects with a higher score for this pattern had lower aspartate aminotransferase (AST), $\gamma$-glutamyl transpeptidase ( $\gamma$-GTP) and UAE values although these findings disappeared in multivariable adjusted model. Subjects with a higher score for "Fish and Meat" pattern were likely to be more female and higher consumption of food intake (Table 3). On the other hand, there were no significant trend in risk factors for CVD among quintiles in both age and gender, and multivariable adjusted model (Table 5). Table 3 also shows the characteristics of the study subjects according to the quintiles of "Noodle and Soup" pattern scores. Subjects with a higher score for this pattern were likely 
Table 3 Characteristics according to quintile categories based on dietary patterns

\begin{tabular}{|c|c|c|c|c|c|c|c|c|c|}
\hline \multirow[t]{2}{*}{ Variable } & \multicolumn{3}{|c|}{$\begin{array}{l}\text { Seaweeds, vegetables, soy products } \\
\text { and mushrooms }\end{array}$} & \multicolumn{3}{|c|}{ Fish and meat } & \multicolumn{3}{|c|}{ Noodle and soups } \\
\hline & Quintile 1 & Quintile 5 & Model 1 & Quintile 1 & Quintile 5 & Model 1 & Quintile 1 & Quintile 5 & Model 1 \\
\hline Age (years) & $53.9 \pm 8.9$ & $59.5 \pm 8.2$ & - & $58.3 \pm 8.6$ & $57.9 \pm 8.9$ & - & $59.1 \pm 7.9$ & $57.6 \pm 8.7$ & - \\
\hline Gender (male) & $92(63.4)$ & $83(57.2)$ & - & $108(74.5)$ & $86(59.3)$ & - & $83(44.4)$ & $133(85.3)$ & - \\
\hline Body mass index $\left(\mathrm{kg} / \mathrm{m}^{2}\right)$ & $25.8 \pm 4.0$ & $24.1 \pm 4.2$ & -1.64 & $24.7 \pm 3.8$ & $24.8 \pm 4.3$ & 0.40 & $23.9 \pm 3.9$ & $25.1 \pm 4.1$ & $3.04^{* *}$ \\
\hline Estimated duration of diabetes (years) & $9.5 \pm 6.8$ & $9.4 \pm 6.4$ & -1.44 & $9.9 \pm 6.8$ & $10.4 \pm 6.9$ & 1.00 & $9.7 \pm 7.3$ & $9.5 \pm 7.0$ & 0.00 \\
\hline MEQ & $55.3 \pm 8.0$ & $58.7 \pm 6.8$ & $2.55^{* * *}$ & $57.6 \pm 7.5$ & $57.6 \pm 7.4$ & -0.11 & $57.9 \pm 7.4$ & $57.5 \pm 7.1$ & -1.21 \\
\hline PSQI & $5.8 \pm 3.8$ & $4.8 \pm 2.6$ & -1.53 & $5.0 \pm 3.1$ & $5.5 \pm 3.4$ & 1.35 & $5.1 \pm 3.4$ & $5.1 \pm 2.8$ & 1.03 \\
\hline BDI-II & $11.4 \pm 7.5$ & $9.1 \pm 7.4$ & $-2.18^{*}$ & $10.0 \pm 7.5$ & $10.8 \pm 8.0$ & 0.71 & $9.8 \pm 8.1$ & $9.6 \pm 7.9$ & 0.47 \\
\hline Energy intake (kcal/day) & $1614 \pm 655$ & $2000 \pm 546$ & $6.43^{* * *}$ & $1621 \pm 506$ & $2154 \pm 658$ & $10.90^{* * *}$ & $1542 \pm 502$ & $2055 \pm 665$ & 7.55 \\
\hline Current smoker (\%) & $34(23.4)$ & $27(18.6)$ & -0.28 & $41(28.3)$ & $27(18.6)$ & -0.64 & $42(22.5)$ & $46(29.5)$ & 0.14 \\
\hline Physical activity (kcal/day) & $37.2 \pm 65.0$ & $51.7 \pm 84.1$ & $2.17^{*}$ & $44.5 \pm 65.1$ & $46.2 \pm 85.6$ & 1.14 & $40.3 \pm 67.9$ & $51.8 \pm 95.2$ & 1.73 \\
\hline Worker (yes) & $114(78.6)$ & $92(63.4)$ & -1.22 & $105(72.4)$ & $105(72.4)$ & 1.31 & 132(70.6) & $118(75.6)$ & -0.84 \\
\hline Sleep duration (hours) & $6.4 \pm 1.4$ & $6.5 \pm 1.2$ & -1.15 & $6.5 \pm 1.3$ & $6.4 \pm 1.3$ & -0.14 & $6.4 \pm 1.3$ & $6.4 \pm 1.1$ & -0.27 \\
\hline Diabetes medication (yes) & $130(89.7)$ & $114(78.6)$ & $-2.50^{*}$ & $122(84.1)$ & $120(82.8)$ & -033 & $155(82.9)$ & $132(84.6)$ & -0.21 \\
\hline Hypertension medication (yes) & $74(51.0)$ & $62(42.8)$ & -1.84 & $71(49.0)$ & $78(53.8)$ & 1.60 & $85(45.5)$ & $74(47.4)$ & -0.03 \\
\hline Hyperlipidemia medication (yes) & $90(62.1)$ & $87(60.0)$ & -0.93 & 89 (61.4) & $86(59.3)$ & -1.10 & $106(56.7)$ & $86(55.1)$ & 1.37 \\
\hline
\end{tabular}

Data are mean \pm SD, median [range: $25 \%$ to $75 \%$ ] or number of subjects (percentage) before adjustment. ${ }^{*} P<0.05,{ }^{* *} P<0.01,{ }^{* * *} P<0.001$

Model 1: Trend estimation for linear trends across quintiles is based on linear regression analysis for continuous variables or logistic regression analysis for categorical variables adjusted for age and gender. Standardized regression coefficients are shown. $B D /$ beck depression inventory, MEQ morningness-eveningness questionnaire, PSQI: Pittsburg sleep quality index

Table 4 Characteristics according to quintile categories in each dietary pattern

\begin{tabular}{|c|c|c|c|c|c|c|c|c|c|}
\hline \multirow[t]{2}{*}{ Variable } & \multicolumn{3}{|c|}{ Meat, fats and oils, seasonings and eggs } & \multicolumn{3}{|c|}{ Fruit, dairy products and sweets } & \multicolumn{3}{|c|}{ Rice and miso soups } \\
\hline & Quintile 1 & Quintile 5 & Model 1 & Quintile 1 & Quintile 5 & Model 1 & Quintile 1 & Quintile 5 & Model 1 \\
\hline Age (years) & $60.6 \pm 6.7$ & $54.9 \pm 8.9$ & - & $56.6 \pm 9.1$ & $58.7 \pm 8.2$ & - & $57.5 \pm 8.8$ & $58.3 \pm 8.6$ & - \\
\hline Gender (male) & $104(55.3)$ & $132(72.9)$ & - & $134(82.7)$ & 109(55.9) & - & $106(55.5)$ & $137(76.5)$ & - \\
\hline Body mass index $\left(\mathrm{kg} / \mathrm{m}^{2}\right)$ & $24.3 \pm 3.9$ & $25.3 \pm 4.5$ & -0.11 & $24.4 \pm 3.8$ & $24.6 \pm 4.2$ & 1.20 & $25.2 \pm 4.3$ & $24.4 \pm 3.8$ & -1.80 \\
\hline Estimated duration of diabetes (years) & $10.0 \pm 7.4$ & $9.5 \pm 6.5$ & 0.47 & $9.4 \pm 7.0$ & $10.2 \pm 7.8$ & 0.76 & $9.5 \pm 6.7$ & $10.2 \pm 7.0$ & 0.83 \\
\hline MEQ & $58.9 \pm 7.4$ & $56.5 \pm 7.4$ & $-2.14^{*}$ & $58.6 \pm 7.8$ & $56.3 \pm 7.2$ & $-3.36^{* * *}$ & $57.1 \pm 7.9$ & $56.9 \pm 7.5$ & -0.91 \\
\hline PSQI & $4.5 \pm 2.6$ & $5.3 \pm 3.1$ & $2.00^{*}$ & $5.1 \pm 3.0$ & $5.2 \pm 3.1$ & 0.02 & $5.2 \pm 2.9$ & $5.0 \pm 2.4$ & -0.06 \\
\hline BDI-II & $9.6 \pm 8.1$ & $10.0 \pm 6.8$ & 0.65 & $9.7 \pm 7.1$ & $10.6 \pm 8.6$ & 1.24 & $10.3 \pm 7.9$ & $10.4 \pm 8.5$ & 0.98 \\
\hline Energy intake (kcal/day) & $1440 \pm 479$ & $2118 \pm 651$ & $12.94^{* * *}$ & $1781 \pm 618$ & $1909 \pm 626$ & $4.14^{* * *}$ & $1418 \pm 456$ & $2047 \pm 568$ & $11.01^{* * *}$ \\
\hline Current smoker (\%) & $39(20.7)$ & $50(27.6)$ & -0.03 & $55(34.0)$ & $32(16.4)$ & $-2.54^{*}$ & $46(24.1)$ & $42(23.5)$ & -1.00 \\
\hline Physical activity (kcal/day) & $39.3 \pm 65.3$ & $39.1 \pm 73.5$ & 0.13 & $46.4 \pm 78.9$ & $45.5 \pm 80.7$ & -0.45 & $41.3 \pm 62.8$ & $47.8 \pm 84.4$ & 1.08 \\
\hline Worker (yes) & $128(68.1)$ & 150(82.9) & 0.11 & $134(82.7)$ & $128(65.6)$ & $-2.51^{*}$ & $131(68.6)$ & $138(77.1)$ & 1.91 \\
\hline Sleep duration (hours) & $6.7 \pm 1.2$ & $6.3 \pm 1.2$ & -1.88 & $6.5 \pm 1.3$ & $6.5 \pm 1.1$ & -0.02 & $6.5 \pm 1.1$ & $6.4 \pm 1.2$ & -0.55 \\
\hline Diabetes medication (yes) & 164(87.2) & $151(83.4)$ & -0.58 & $140(86.4)$ & $164(84.1)$ & -1.12 & $167(87.4)$ & $153(85.5)$ & -0.80 \\
\hline Hypertension medication (yes) & $92(48.9)$ & $82(45.3)$ & -0.75 & $95(58.6)$ & $82(42.1)$ & $-2.86^{* *}$ & $94(49.2)$ & $95(53.1)$ & 0.01 \\
\hline Hyperlipidemia medication (yes) & 122(64.9) & $99(54.7)$ & -0.35 & $78(48.1)$ & $131(67.2)$ & $2.86^{* *}$ & $118(61.8)$ & $95(53.1)$ & -1.14 \\
\hline
\end{tabular}

Data are mean \pm SD, median [range: $25 \%$ to $75 \%$ ] or number of subjects (percentages) before adjustment. ${ }^{*} P<0.05$, ${ }^{* *} P<0.01,{ }^{* * *} P<0.001$

Model 1: Trend estimation for linear trends across quintiles is based on linear regression analysis for continuous variables or logistic regression analysis for categorical variables adjusted for age and gender. Standardized regression coefficients are shown. See Table 3 for abbreviations 
Table 5 Cardio-renal-metabolic parameters according to quintile categories in each dietary pattern

\begin{tabular}{|c|c|c|c|c|c|c|c|c|c|c|c|c|}
\hline \multirow[t]{2}{*}{ Variable } & \multicolumn{4}{|c|}{ Seaweeds, vegetables, soy products and mushrooms } & \multicolumn{4}{|c|}{ Fish and meat } & \multicolumn{4}{|c|}{ Noodle and soup } \\
\hline & Quintile 1 & Quintile 5 & Model 1 & Model 2 & Quintile 1 & Quintile 5 & Model 1 & Mode I 2 & Quintile 1 & Quintile 5 & Model 1 & Model 2 \\
\hline AST (U/L) & $22[19,29]$ & $22[17,26]$ & $-2.07^{*}$ & -1.71 & $21[18,27]$ & $23[19,28]$ & 1.14 & 0.98 & $21[17,25]$ & $23[18,29]$ & $3.25^{* *}$ & $2.60^{*}$ \\
\hline ALT (U/L) & $24[17,41]$ & $22[16,33]$ & -1.52 & -0.80 & $23[16,34]$ & $25[17,34]$ & 0.94 & 0.69 & $19[15,30]$ & $26[18,39]$ & $4.17^{* * *}$ & $3.29^{*}$ \\
\hline Y-GTP (U/L) & $28[18,48]$ & $22[15,36]$ & $-2.46^{*}$ & -1.95 & $25[18,44]$ & $25[18,38]$ & 0.13 & -0.01 & $22[16,30]$ & $30[21,50]$ & $3.62^{* * *}$ & $2.75^{* *}$ \\
\hline Uric Acid (mg/dl) & $5.5 \pm 1.2$ & $5.4 \pm 1.2$ & 0.12 & 0.51 & $5.6 \pm 1.2$ & $5.4 \pm 1.2$ & 0.75 & 0.63 & $5.2 \pm 1.2$ & $5.8 \pm 1.2$ & 0.85 & 0.27 \\
\hline eGFR (ml/min/ $\left.1.73 \mathrm{~m}^{2}\right)$ & $81 \pm 18$ & $77 \pm 16$ & - & 0.17 & $77 \pm 18$ & $78 \pm 17$ & - & 0.08 & $78 \pm 19$ & $79 \pm 17$ & - & -0.39 \\
\hline Total cholesterol (mg/dl) & $190 \pm 27$ & $186 \pm 29$ & -1.64 & -1.70 & $184 \pm 30$ & $187 \pm 27$ & 0.16 & 0.09 & $188 \pm 30$ & $185 \pm 27$ & 0.39 & 0.22 \\
\hline HDL-C (mg/dl) & $59 \pm 14$ & $62 \pm 14$ & 1.08 & 0.60 & $58 \pm 13$ & $59 \pm 14$ & -0.03 & -0.07 & $62 \pm 15$ & $57 \pm 13$ & -1.39 & -0.59 \\
\hline Triglycerides (mg/dl) & $101[71,163]$ & $96[64,144]$ & -1.74 & -1.06 & $94[66,144]$ & $112[67,152]$ & 0.88 & 0.78 & $89[64,135]$ & $116[78,164]$ & $2.82^{* *}$ & 1.73 \\
\hline $\begin{array}{l}\text { Fasting blood glucose } \\
(\mathrm{mg} / \mathrm{dl})\end{array}$ & $137 \pm 34$ & $131 \pm 27$ & 0.01 & 0.49 & $133 \pm 32$ & $138 \pm 35$ & 1.01 & 0.81 & $132 \pm 31$ & $137 \pm 30$ & 0.72 & 0.43 \\
\hline $\mathrm{HbA1c}$ & $7.1 \pm 1.1$ & $6.9 \pm 1.1$ & -0.48 & -0.04 & $6.9 \pm 0.9$ & $7.1 \pm 1.1$ & 1.02 & 0.95 & $6.9 \pm 1.0$ & $7.0 \pm 1.0$ & 1.66 & 1.21 \\
\hline Systolic BP(mmHg) & $128 \pm 13$ & $127 \pm 15$ & 0.12 & 0.83 & $127 \pm 14$ & $126 \pm 15$ & 0.09 & -0.10 & $126 \pm 15$ & $128 \pm 13$ & 1.83 & 0.92 \\
\hline Diastolic BP (mmHg) & $79 \pm 10$ & $75 \pm 10$ & -1.00 & -0.53 & $78 \pm 13$ & $76 \pm 11$ & -0.19 & -0.33 & $75 \pm 14$ & $78 \pm 10$ & 0.99 & 0.27 \\
\hline UAE (mg/g creatinine) & $10[6,21]$ & $10[5,17]$ & $-2.12^{*}$ & -1.74 & $10[6,24]$ & $10[7,23]$ & -0.80 & -0.88 & $11[6,30]$ & $9[6,19]$ & -0.39 & -1.18 \\
\hline baPWV (cm/s) & $1528 \pm 294$ & $1556 \pm 262$ & -1.62 & -1.53 & $1536 \pm 258$ & $1535 \pm 248$ & 0.79 & 0.72 & $1549 \pm 288$ & $1536 \pm 262$ & -0.91 & -0.94 \\
\hline
\end{tabular}

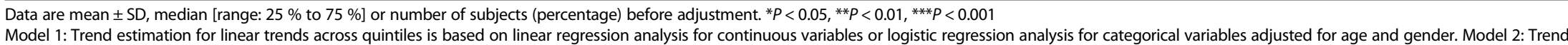
Model 1: Trend estimation for linear trends across quintiles is based on linear regression analysis for continuous variables or logistic regression analysis for categorical variables adjusted for age and gender. Model 2: Trend
estimation for linear trends across quintiles is based on linear regression analysis for continuous variables or logistic regression analysis for categorical variables adjusted for age, gender, BMI, morningness-eveningness questionnaire, Pittsburg Sleep Quality Index, Beck Depression inventory, current smoking, and physical activity. Standardized regression coefficients are shown. ALT alanine aminotransferase, AST aspartate aminotransferase, baPWV brachial-ankle pulse wave velocity, BP blood pressure, eGFR estimated glomerular filtration rate, HDL-C high-density lipoprotein-cholesterol, UAE urinary albumin excretion, $\gamma$-GTP $\gamma$-glutamyl transpeptidase 
Table 6 Cardio-renal-metabolic parameters according to quintile categories in each dietary pattern

\begin{tabular}{|c|c|c|c|c|c|c|c|c|c|c|c|c|}
\hline \multirow[t]{2}{*}{ Variable } & \multicolumn{4}{|c|}{ Meat, fats and oils, seasonings and eggs } & \multicolumn{4}{|c|}{ Fruit, dairy products and sweets } & \multicolumn{4}{|c|}{ Rice and miso soups } \\
\hline & Quintile 1 & Quintile 5 & Model 1 & Model 2 & Quintile 1 & Quintile 5 & Model 1 & Model 2 & Quintile 1 & Quintile 5 & Model 1 & Model 2 \\
\hline AST (U/L) & $22[19,27]$ & $21[17,26]$ & -1.14 & -1.15 & $22[18,28]$ & $21[18,27]$ & 0.49 & 0.09 & $21[17,27]$ & $22[18,27]$ & 1.05 & 1.45 \\
\hline ALT (U/L) & $21[16,31]$ & $24[17,35]$ & -0.40 & -0.49 & $22[16,33]$ & $23[17,34]$ & $2.58^{*}$ & $2.10^{*}$ & $22[15,35]$ & $24[17,34]$ & 0.92 & 1.48 \\
\hline$\gamma$-GTP (U/L) & $25[16,36]$ & $27[17,46]$ & -1.19 & -1.33 & $32[20,56]$ & $24[17,36]$ & $-3.51^{* * *}$ & $-4.20^{* * *}$ & $26[17,38]$ & $26[19,42]$ & 0.11 & 0.69 \\
\hline Uric Acid (mg/dl) & $5.4 \pm 1.2$ & $5.5 \pm 1.2$ & $-2.27^{*}$ & $-2.31^{*}$ & $5.8 \pm 1.3$ & $5.3 \pm 1.2$ & -0.58 & -1.16 & $5.4 \pm 1.3$ & $5.6 \pm 1.1$ & 0.23 & 0.46 \\
\hline $\mathrm{eGFR}\left(\mathrm{ml} / \mathrm{min} / 1.73 \mathrm{~m}^{2}\right)$ & $75 \pm 15$ & $80 \pm 19$ & - & 1.24 & $78 \pm 17$ & $79 \pm 19$ & - & -0.56 & $79 \pm 19$ & $77 \pm 17$ & - & -0.87 \\
\hline Total cholesterol (mg/dl) & $185 \pm 26$ & $188 \pm 30$ & 0.79 & 0.83 & $185 \pm 27$ & $185 \pm 29$ & -1.04 & -1.11 & $188 \pm 29$ & $183 \pm 29$ & -0.26 & -0.09 \\
\hline $\mathrm{HDL}-\mathrm{C}(\mathrm{mg} / \mathrm{dl})$ & $59 \pm 14$ & $59 \pm 16$ & 1.25 & 1.35 & $61 \pm 14$ & $60 \pm 14$ & $-2.87^{* *}$ & $-2.65^{* *}$ & $59 \pm 15$ & $58 \pm 13$ & -0.50 & -1.05 \\
\hline Triglycerides (mg/dl) & $98[68,144]$ & $112[72,157]$ & -0.14 & -0.27 & $112[70,158]$ & $98[71,143]$ & -0.45 & -0.99 & $99[71,148]$ & $100[70,158]$ & -0.07 & 0.70 \\
\hline Fasting blood glucose (mg/dl) & $130 \pm 30$ & $137 \pm 33$ & 0.93 & 0.64 & $136 \pm 30$ & $130 \pm 31$ & -1.22 & -1.74 & $135 \pm 33$ & $136 \pm 31$ & 0.28 & 0.21 \\
\hline $\mathrm{HbA1c}$ & $6.8 \pm 0.9$ & $7.0 \pm 1.0$ & 1.55 & 1.25 & $6.8 \pm 1.0$ & $6.9 \pm 0.9$ & 0.56 & 0.12 & $7.0 \pm 1.1$ & $7.0 \pm 0.9$ & 0.65 & 0.79 \\
\hline Systolic BP(mmHg) & $126 \pm 15$ & $127 \pm 13$ & 0.37 & 0.22 & $128 \pm 13$ & $125 \pm 14$ & $-2.65^{* *}$ & $-3.01^{* *}$ & $128 \pm 15$ & $127 \pm 13$ & -0.47 & 0.10 \\
\hline Diastolic BP (mmHg) & $76 \pm 10$ & $79 \pm 13$ & -0.37 & -0.44 & $80 \pm 11$ & $76 \pm 12$ & -1.89 & $-2.31^{*}$ & $77 \pm 11$ & $78 \pm 13$ & 1.19 & 1.61 \\
\hline UAE (mg/g creatinine) & $10[6,24]$ & $10[5,24]$ & -0.17 & -0.28 & $12[7,36]$ & $9[6,18]$ & $-3.20^{* *}$ & $-3.44^{* * *}$ & $10[6,23]$ & $11[6,23]$ & -1.23 & -0.77 \\
\hline baPWV (cm/s) & $1577 \pm 282$ & $1508 \pm 265$ & -0.07 & -0.33 & $1574 \pm 314$ & $1530 \pm 259$ & $-3.04^{* *}$ & $-3.14^{* *}$ & $1536 \pm 256$ & $1556 \pm 277$ & 0.18 & 0.15 \\
\hline
\end{tabular}

Data are mean \pm SD, median [range: $25 \%$ to $75 \%$ ] or number of subjects (proportion) before adjustment. ${ }^{*} P<0.05$, ** $P<0.01, * * * P<0.001$

Model 1: Trend estimation for linear trends across quintiles is based on linear regression analysis for continuous variables or logistic regression analysis for categorical variables adjusted for age and gender. Model 2: Trend

estimation for linear trends across quintiles is based on linear regression analysis for continuous variables or logistic regression analysis for categorical variables adjusted for age, gender, BMI, morningness-eveningness

questionnaire, Pittsburg Sleep Quality Index, Beck Depression inventory, current smoking, and physical activity. Standardized regression coefficients are shown. See Table 5 for abbreviations 
to be males with higher BMI and consumption of food intake. Furthermore, patients with a higher score of this pattern had significantly higher AST, alanine aminotransferase (ALT) levels and $\gamma$-GTP compared to those with a lower score in both age- and gender-adjusted model, and multivariate adjusted model (Table 5). Furthermore, patients with a higher score of this pattern had significantly higher triglyceride levels compared to those with a lower score in the age- and gender-adjusted model.

Table 4 shows the characteristics of the study subjects according to the quintiles of "Meat, Fats and oils, Seasonings and Eggs" pattern scores in age- and genderadjusted model. Subjects with a higher score for this pattern were younger males with lower score of MEQ, higher score of PSQI and higher consumption of food intake. On the other hand, there were no significant trend in risk factors for CVD except uric acid in both age- and gender-adjusted model and multivariable adjusted model (Table 6). Table 4 also displays the characteristics of the study subjects according to the quintiles of "Fruit, Dairy products and Sweets" pattern scores. This pattern was also characterized by lower consumption of alcohol. Subjects with a higher score for this pattern were more likely non-worker, non-smoker females with higher consumption of food intake, with lower score of MEQ. Lower use of hypertension medication and higher use of hyperlipidemia mediation were found in the highest quintile. Regarding risk factors for CVD, patients with higher a score of this pattern had lower $\gamma$ GTP level, HDL level, systolic BP, UAE and baPWV but slightly higher ALT level compared to those with a lower score in age- and gender-adjusted model, and multivariate adjusted model (Table 6). Table 4 shows the characteristics of the study subjects according to the quintiles of "Rice and Miso soups" pattern scores. Subjects with a higher score for this pattern were higher consumption of food while there was no significant trend in risk factors for CVD.

\section{Discussion}

Among the six dietary patterns classified in this study, the "Seaweeds, Vegetables, Soy products and Mushrooms" pattern was associated with lower use of diabetes medications and healthier lifestyles. "Noodle and soup" pattern was associated with higher BMI, higher AST, ALT, $\gamma$-GTP and triglyceride levels. "Fruit, Dairy products and Sweets" pattern was associated with lower $\gamma$ GTP levels, BP, UAE and baPWV. On the other hand, the remaining three dietary patterns did not negatively affect risk factors for CVD.

Subjects with a higher score of "Seaweeds, Vegetables, Soy products and Mushrooms" pattern had healthier lifestyles, such as more morning type, less depressive symptoms and higher physical activity. Even after adjustment for those factors, the "Seaweeds, Vegetables, Soy products and Mushroom" pattern was associated with lower use of diabetes medication (data not shown), while there was no significant trend of $\mathrm{HbA1c}$ among their quintile categories. It is possible that the "Seaweeds, Vegetables, Soy products and Mushrooms" pattern positively affect glucose metabolism even in patients with T2DM, since a dietary pattern characterized by high consumption of vegetables, soy products and seaweed was reported to be inversely associated with lower incidence of T2DM [12-14] in the general population. Fiber [8], antioxidant vitamins [34], magnesium [6,8] and phytoestrogens [35], which were abundant in those foods, have been reported to improve glucose metabolism and reduce insulin resistance. In addition, one previous study demonstrated that a healthy Japanese dietary pattern characterized by high consumption of vegetables, fruit, mushrooms and soy products was associated with fewer depressive symptoms in municipal employees [36]. Consistent with this finding, the almost similar type of "Seaweeds, Vegetables, Soy products and Mushrooms" pattern defined in this study was also associated with lower prevalence of depressive symptoms in patients with T2DM. Generally, vegetables and mushrooms contain vitamins and folate, which may reduce the prevalence of depression. In fact, it was demonstrated that antioxidant vitamins may protect neuropsychiatric disorders [37]. Similarly, folate intake was reported to be reduce depressive symptoms through metabolism of monoamines like serotonin and homocysteine in the brain [38]. Taken together, it seems that "Seaweeds, Vegetables, Soy products and Mushroom" dietary pattern is beneficial for patients with T2DM.

The "Fish and Meat" pattern was not associated with risk factors for CVD in this study. It was demonstrated that similar dietary pattern was also associated with the prevalence of the metabolic syndrome in Western populations [39] but not in Japanese workers [40]. These distinct results may be associated with relatively lower intake of meat and higher consumption of fish, vegetable and fruit in Japanese people compared to those in Western populations.

A higher score of "Noodle and Soup" pattern was associated with obesity characterized by high BMI, high AST, ALT, $\gamma$-GTP and triglyceride levels. Noodles including Japanese noodles, Chinese style noodles and pasta are one of the most favorite dishes in Japan. Higher intake of noodles, which contains high carbohydrate, may lead to obesity and fatty liver. Higher intake of oil included in the soup may be also associated with obesity and fatty liver.

We found that "Meat, Fats and Oils, Seasonings and Eggs" pattern represented by high intake of meat, fats and oils, seasonings and eggs was not related with risk factors for CVD, except high uric acid levels. On the other hand, a recent study reported that a similar dietary 
pattern, named high-fat pattern, was inversely associated with $\mathrm{HbA1c}$ levels in Japanese non-T2DM population [41]. The difference between our study and the above could be related to a number of differences among subjects, such as T2DM or non-T2DM, obesity, fat-derived energy intake and other dietary components. While frequent intake of meat, processed meat and eggs negatively affect lipid metabolism, "Meat, Fats and Oils, Seasonings and Eggs" pattern was not associated with lipid metabolism in this study. This may be related to the relatively higher intake of vegetables in addition to frequent intake of meat, processed meat and eggs. Indeed, it was reported that vegetable-rich diet seems to reduce cholesterol concentrations and other cardiovascular risk factors in patients with T2DM as effectively as in patients with non-T2DM [42].

The "Fruit, Dairy products and Sweets" pattern with less frequent consumption of alcohol and cigarette seemed to be preferred by females. Especially, this pattern was associated with lower BP, UAE and baPWV, even after adjustment for multivariate factors. These data may be reasonable because frequent dietary fruit intake was shown to be associated with lower $\mathrm{BP}$ and reduced atherosclerotic changes [43]. Also, previous studies demonstrated favorable preventive effects of dairy product on the incidence of hypertension, atherosclerosis and T2DM [44]. Antioxidant nutrients, such as vitamin $\mathrm{C}$ and $\mathrm{E}$, carotene and dietary fiber are abundant in fruit. Similarly, dairy products contain calcium, magnesium, potassium, and vitamin D. These nutrients may have beneficial roles on risk factors for CVD in this pattern despite the co-presence of unhealthy diet pattern such as higher sweet consumption.

Recent studies showed that high consumption of rice contributed to the onset of T2DM in Chinese [9] and Japanese women, but not Japanese men [45]. While the mechanism by which frequent intake of rice increases the risk of T2DM remains largely unknown, the high glycemic index of white rice may affect glucose metabolism [46]. However, the "Rice and Soups" pattern was not associated with glycemic control in our patients with T2DM. Considering that subjects with high scores of this pattern showed higher consumption of fiber (data not shown), they may consume brown rice, which contains more dietary fiber, vitamin and minerals compared with white rice or fiber-rich foods with rice.

The present study has certain limitations. First, the cross-sectional design does not allow inference of a causal relationships between dietary patterns and risk factors for CVD. In addition, we could not confirm that the participating subjects adhered to the same dietary pattern throughout life. Especially, dietary patterns probably change depending on many factors such as status of glycemic control (good glycemic control vs. poor glycemic control) and estimated duration of T2DM (shorter duration vs. longer duration). This may cause reverse causality. Thus, it is not reasonable to conclude that our data could be generalized to a wider range of population, even in Japanese patients with T2DM. Second, we evaluated dietary pattern by self-reported questionnaires, though this method has been widely used in many studies. The results may be influenced by social desirability and recall bias. In addition, we evaluated only 56 food and beverage items. This could be associated with incomplete list of dietary patterns and an underestimation of energy intake. Finally, our results may be affected by several limiting features of factor analysis. It is not possible for investigators to avoid making judgmental decision at their discretion [47]. The results are dependent on the criterion to determine the number of factors to be retained and, the methods for rotation of factor axes and for labeling the dietary pattern. Therefore, our data should be interpreted with caution. For example, other remaining dietary patters or other remaining food items in each dietary pattern may be associated with risk factors for CVD.

\section{Conclusion}

Our study showed the relationship between dietary patterns and risk factors for CVD in T2DM patients without history of CVD. Thus, dietary pattern could be a potentially important therapeutic target to achieve appropriate metabolic control and prevent the onset of future CVD in patients with T2DM.

\section{Abbreviations}

ALT: alanine aminotransferase; AST: aspartate aminotransferase; BaPWV: brachial-ankle pulse wave velocity; BDI: beck depression inventory; BDHQ: brief, self-administered diet history questionnaire; BMl: body mass index; BP: blood pressure; CVD: cardiovascular disease; eGFR: estimated glomerular filtration rate; $\gamma$-GTP: $\gamma$-glutamyl transpeptidase; HDL: high-density lipoprotein-cholesterol; IPAQ: International physical activity questionnaire; MEQ: morningness-eveningness questionnaire; PSQI: Pittsburg sleep quality index; T2DM: type 2 diabetes mellitus; UAE: urinary albumin excretion.

\section{Competing interests \\ T.M. received research grand from Manpei Suzuki Diabetes Foundation. H.W. received lecture fees from Boehringer Ingelheim, Sanofi-Aventis, Ono Pharmaceutical Co., Novo Nordisk Pharma, Novartis Pharmaceuticals, Eli Lilly, Sanwakagaku Kenkyusho, Daiichi Sankyo Inc., Takeda Pharmaceutical Co., MSD, Dainippon Sumitomo Pharm., Kowa Co. and research funds from Boehringer Ingelheim, Pfizer, Mochida Pharmaceutical Co., Sanofi-Aventis, Novo Nordisk Pharma, Novartis Pharmaceuticals, Sanwakagaku Kenkyusho, Terumo Corp. Eli Lilly, Mitsubishi Tanabe Pharma, Daiichi Sankyo Inc., Takeda Pharmaceutical Co., MSD, Shionogi, Pharma, Dainippon Sumitomo Pharma, Kissei Pharma, and Astrazeneca.}

\section{Authors' contributions}

All authors contribute to the study design and were involved at all stages of manuscript development. YO and TM mainly drafted the manuscript. MG, a statistician, contributed to analysis of research data. All authors were also involved in analysis and interpretation of data, reviewed/edited the manuscript and approved the final manuscript. HW is the principal guarantors of this work and has full access to all study data and takes responsibility for the integrity of the data and accuracy of data analysis. All authors have read and agreed to the publication of the manuscript. 
The manuscript has not been submitted elsewhere. All authors read and approved the final manuscript.

\section{Acknowledgments}

This study was supported by research grand from Manpei Suzuki Diabetes Foundation (to T.M.). The authors thank the following staff who participated in this trial, including Mses Risa Yamamoto and Emi Ito (Department of Metabolism and Endocrinology, Juntendo University Graduate School of Medicine), Satako Douguchi, Chiyoko Sato, Yoko Ono, Norie Shiina, Nana Shiozawa, Mariko Kobori, Misato Ojima, Akiko Haginoya, and Kumiko Fujisaku, and Messrs Tadanori Koibuchi and Katuhiro Kawauchi (Naka Memorial Clinic) for the excellent technical support.

\section{Author details}

1 Department of Metabolism \& Endocrinology, Juntendo University Graduate School of Medicine, 2-1-1 Hongo, Bunkyoku, Tokyo 113-8421, Japan. ${ }^{2}$ Center for Molecular Diabetology, Juntendo University Graduate School of Medicine, Bunkyoku, Tokyo 113-8421, Japan. ${ }^{3}$ Center for Therapeutic Innovations in Diabetes, Juntendo University Graduate School of Medicine, Bunkyoku, Tokyo 113-8421, Japan. ${ }^{4}$ Sportology Center, Juntendo University Graduate School of Medicine, Bunkyoku, Tokyo 113-8421, Japan. ${ }^{5}$ Naka Memorial Clinic, 745-5, Nakadai, Naka, Ibaraki 311-0113, Japan. ${ }^{6}$ Department of Clinical Trial and Clinical Epidemiology, Faculty of Medicine, University of Tsukuba, 1-1-1, Tennodai, Tsukuba, Ibaraki 305-8575, Japan.

Received: 7 July 2015 Accepted: 21 January 2016 Published online: 04 February 2016

\section{References}

1. Knowler WC, Barrett-Connor E, Fowler SE, Hamman RF, Lachin JM, Walker EA, et al. Reduction in the incidence of type 2 diabetes with lifestyle intervention or metformin. N Engl J Med. 2002;346(6):393-403. doi:10.1056/NEJMoa012512.

2. Sone H, Tanaka S, limuro S, Tanaka S, Oida K, Yamasaki Y, et al. Long-term lifestyle intervention lowers the incidence of stroke in Japanese patients with type 2 diabetes: a nationwide multicentre randomised controlled trial (the Japan Diabetes Complications Study). Diabetologia. 2010;53(3):419-28. doi:10.1007/s00125-009-1622-2.

3. Hu G, Jousilahti P, Barengo NC, Qiao Q, Lakka TA, Tuomilehto J. Physical activity, cardiovascular risk factors, and mortality among Finnish adults with diabetes. Diabetes Care. 2005;28(4):799-805.

4. Look ARG, Wing RR, Bolin P, Brancati FL, Bray GA, Clark JM, et al. Cardiovascular effects of intensive lifestyle intervention in type 2 diabetes. N Engl J Med. 2013;369(2):145-54. doi:10.1056/NEJMoa1212914.

5. Ford ES, Mokdad AH. Fruit and vegetable consumption and diabetes mellitus incidence among U.S. adults. Prev Med. 2001;32(1):33-9. doi:10.1006/pmed.2000.0772.

6. Song Y, Manson JE, Buring JE, Liu S. Dietary magnesium intake in relation to plasma insulin levels and risk of type 2 diabetes in women. Diabetes Care. 2004:27(1):59-65.

7. Fumeron F, Lamri A, Abi Khalil C, Jaziri R, Porchay-Balderelli I, Lantieri O, et al. Dairy consumption and the incidence of hyperglycemia and the metabolic syndrome: results from a french prospective study, data from the epidemiological study on the insulin resistance syndrome (DESIR). Diabetes Care. 2011;34(4):813-7. doi:10.2337/dc10-1772

8. Schulze MB, Schulz M, Heidemann C, Schienkiewitz A, Hoffmann K, Boeing H. Fiber and magnesium intake and incidence of type 2 diabetes: a prospective study and meta-analysis. Arch Intern Med. 2007;167(9):956-65. doi:10.1001/archinte.167.9.956

9. Villegas R, Liu S, Gao YT, Yang G, Li H, Zheng W, et al. Prospective study of dietary carbohydrates, glycemic index, glycemic load, and incidence of type 2 diabetes mellitus in middle-aged Chinese women. Arch Intern Med. 2007;167(21):2310-6. doi:10.1001/archinte.167.21.2310.

10. Liu S, Manson JE, Stampfer MJ, Hu FB, Giovannucci E, Colditz GA, et al. A prospective study of whole-grain intake and risk of type 2 diabetes mellitus in US women. Am J Public Health. 2000;90(9):1409-15.

11. Hu FB. Dietary pattern analysis: a new direction in nutritional epidemiology. Curr Opin Lipidol. 2002;13(1):3-9.

12. Montonen J, Knekt P, Harkanen T, Jarvinen R, Heliovaara M, Aromaa A, et al. Dietary patterns and the incidence of type 2 diabetes. Am J Epidemiol. 2005;161(3):219-27. doi:10.1093/aje/kwi039.
13. Odegaard AO, Koh WP, Butler LM, Duval S, Gross MD, Yu MC, et al. Dietary patterns and incident type 2 diabetes in chinese men and women: the singapore chinese health study. Diabetes Care. 2011;34(4):880-5. doi:10.2337/dc10-2350.

14. Morimoto A, Ohno Y, Tatsumi Y, Mizuno S, Watanabe S. Effects of healthy dietary pattern and other lifestyle factors on incidence of diabetes in a rural Japanese population. Asia Pac J Clin Nutr. 2012;21(4):601-8.

15. Cho YA, Kim J, Cho ER, Shin A. Dietary patterns and the prevalence of metabolic syndrome in Korean women. Nutrition, metabolism, and cardiovascular diseases: NMCD. 2011;21(11):893-900. doi:10.1016/..numecd.2010.02.018.

16. Nettleton JA, Steffen LM, Schulze MB, Jenny NS, Barr RG, Bertoni AG, et al. Associations between markers of subclinical atherosclerosis and dietary patterns derived by principal components analysis and reduced rank regression in the Multi-Ethnic Study of Atherosclerosis (MESA). The American journal of clinical nutrition. 2007:85(6):1615-25.

17. Shimazu T, Kuriyama S, Hozawa A, Ohmori K, Sato Y, Nakaya N, et al. Dietary patterns and cardiovascular disease mortality in Japan: a prospective cohort study. Int J Epidemiol. 2007;36(3):600-9. doi:10.1093/ije/dym005.

18. van Dam RM, Rimm EB, Willett WC, Stampfer MJ, Hu FB. Dietary patterns and risk for type 2 diabetes mellitus in U.S. men. Ann Intern Med. 2002;136(3):201-9.

19. Fung TT, Schulze M, Manson JE, Willett WC, Hu FB. Dietary patterns, meat intake, and the risk of type 2 diabetes in women. Arch Intern Med. 2004;164(20):2235-40. doi:10.1001/archinte.164.20.2235.

20. Lutsey PL, Steffen LM, Stevens J. Dietary intake and the development of the metabolic syndrome: the atherosclerosis risk in communities study. Circulation. 2008;117(6):754-61. doi:10.1161/CIRCULATIONAHA.107.716159.

21. Murray AE, McMorrow AM, O'Connor E, Kiely C, Mac Ananey O, O'Shea D, et al. Dietary quality in a sample of adults with type 2 diabetes mellitus in Ireland; a cross-sectional case control study. Nutr J. 2013;12:110. doi:10.1186/1475-2891-12-110.

22. Lim JH, Lee YS, Chang HC, Moon MK, Song Y. Association between dietary patterns and blood lipid profiles in Korean adults with type 2 diabetes. J Korean Med Sci. 2011;26(9):1201-8. doi:10.3346/jkms.2011.26.9.1201.

23. Hsu CC, Jhang HR, Chang WT, Lin CH, Shin SJ, Hwang SJ, et al. Associations between dietary patterns and kidney function indicators in type 2 diabetes. Clin Nutr. 2014;33(1):98-105. doi:10.1016/j.clnu.2013.04.010.

24. Osonoi Y, Mita T, Osonoi T, Saito M, Tamasawa A, Nakayama S, et al. Morningness-eveningness questionnaire score and metabolic parameters in patients with type 2 diabetes mellitus. Chronobiol Int. 2014;31(9):1017-23. doi:10.3109/07420528.2014.943843.

25. Agency SaT. Standard Tables of Food Composition in Japan (in Japanese). 5th ed. Tokyo: Printing Bureau of the Ministry of Finance; 2005. doi:10.1007/s11136-007-9172-x

26. Hone JA, Ostberg $\mathrm{O}$. A self-assessment questionnaire to determine morningness-eveningness in human circadian rhythms. Int J Chronobiol. 1976:4:97-110. doi:10.1001/archinte.166.16.1768.

27. Buysse DJ, Reynolds 3rd CF, Monk TH, Berman SR, Kupfer DJ. The Pittsburgh sleep quality index: a new instrument for psychiatric practice and research. Psychiatry Res. 1989;28(2):193-213.

28. Backhaus J, Junghanns K, Broocks A, Riemann D, Hohagen F. Test-retest reliability and validity of the Pittsburgh sleep quality index in primary insomnia. J Psychosom Res. 2002:53(3):737-40.

29. Beck AT, Steer RA, Brown GK. Manual for the Beck Depression Inventory-II. San Antonio, TX: Psychological Corporation; 1996. doi:10.1207/s15327752jpa6703_13.

30. Craig $C L$, Marshall AL, Sjostrom M, Bauman AE, Booth ML, Ainsworth BE, et al. International physical activity questionnaire: 12-country reliability and validity. Med Sci Sports Exerc. 2003;35(8):1381-95. doi:10.1249/01.MSS.0000078924.61453.FB.

31. Takeno K, Mita T, Nakayama S, Goto H, Komiya K, Abe H, et al. Masked hypertension, endothelial dysfunction, and arterial stiffness in type 2 diabetes mellitus: a pilot study. Am J Hypertens. 2012;25(2):165-70. doi:10.1038/ajh.2011.158.

32. Yamashina A, Tomiyama H, Takeda K, Tsuda H, Arai T, Hirose K, et al. Validity, reproducibility, and clinical significance of noninvasive brachial-ankle pulse wave velocity measurement. Hypertension research : official journal of the Japanese Society of Hypertension. 2002;25(3):359-64.

33. Slattery ML, Boucher KM, Caan BJ, Potter JD, Ma KN. Eating patterns and risk of colon cancer. Am J Epidemiol. 1998;148(1):4-16.

34. Garcia-Bailo B, El-Sohemy A, Haddad PS, Arora P, Benzaied F, Karmali M, et al. Vitamins D, C, and $\mathrm{E}$ in the prevention of type 2 diabetes mellitus: modulation of inflammation and oxidative stress. Biologics: targets \& therapy. 2011:5:7-19. doi:10.2147/BTT.S14417. 
35. Bhathena SJ, Velasquez MT. Beneficial role of dietary phytoestrogens in obesity and diabetes. The American journal of clinical nutrition. 2002;76(6):1191-201.

36. Nanri A, Kimura Y, Matsushita Y, Ohta M, Sato M, Mishima N, et al. Dietary patterns and depressive symptoms among Japanese men and women. Eur J Clin Nutr. 2010;64(8):832-9. doi:10.1038/ejcn.2010.86.

37. Bilici M, Efe H, Koroglu MA, Uydu HA, Bekaroglu M, Deger O. Antioxidative enzyme activities and lipid peroxidation in major depression: alterations by antidepressant treatments. J Affect Disord. 2001;64(1):43-51.

38. Bottiglieri T. Homocysteine and folate metabolism in depression. Prog Neuro-Psychopharmacol Biol Psychiatry. 2005;29(7):1103-12. doi:10.1016/.jpnpbp.2005.06.021

39. Denova-Gutierrez E, Castanon S, Talavera JO, Gallegos-Carrillo K, Flores M, Dosamantes-Carrasco D, et al. Dietary patterns are associated with metabolic syndrome in an urban Mexican population. The Journal of nutrition. 2010;140(10):1855-63. doi:10.3945/jn.110.122671.

40. Akter S, Nanri A, Pham NM, Kurotani K, Mizoue T. Dietary patterns and metabolic syndrome in a Japanese working population. Nutrition \& metabolism. 2013;10(1):30. doi:10.1186/1743-7075-10-30.

41. Nanri A, Mizoue T, Yoshida D, Takahashi R, Takayanagi R. Dietary patterns and A1C in Japanese men and women. Diabetes Care. 2008;31(8):1568-73. doi:10.2337/dc08-0297.

42. Pischke CR, Weidner G, Elliott-Eller M, Scherwitz L, Merritt-Worden TA, Marlin R, et al. Comparison of coronary risk factors and quality of life in coronary artery disease patients with versus without diabetes mellitus. Am J Cardiol. 2006;97(9):1267-73. doi:10.1016/..amjcard.2005.11.051.

43. Chan HT, Yiu KH, Wong CY, Li SW, Tam S, Tse HF. Increased dietary fruit intake was associated with lower burden of carotid atherosclerosis in Chinese patients with Type 2 diabetes mellitus. Diabetic medicine: a journal of the British Diabetic Association. 2013;30(1):100-8. do::10.1111/j.1464-5491.2012.03764.x

44. Chrysant SG, Chrysant GS. An update on the cardiovascular pleiotropic effects of milk and milk products. J Clin Hypertens. 2013;15(7):503-10. doi:10.1111/jch.12110.

45. Nanri A, Mizoue T, Noda M, Takahashi Y, Kato M, Inoue M, et al. Rice intake and type 2 diabetes in Japanese men and women: the Japan public health center-based prospective study. The American journal of clinical nutrition. 2010;92(6):1468-77. doi:10.3945/ajen.2010.29512.

46. Foster-Powell K, Holt SH, Brand-Miller JC. International table of glycemic index and glycemic load values: 2002. The American journal of clinical nutrition. 2002;76(1):5-56.

47. Martinez ME, Marshall JR, Sechrest L. Invited commentary: factor analysis and the search for objectivity. Am J Epidemiol. 1998;148(1):17-9.

\section{Submit your next manuscript to BioMed Central and we will help you at every step:}

- We accept pre-submission inquiries

- Our selector tool helps you to find the most relevant journal

- We provide round the clock customer support

- Convenient online submission

- Thorough peer review

- Inclusion in PubMed and all major indexing services

- Maximum visibility for your research

Submit your manuscript at www.biomedcentral.com/submit

C Biomed Central 